Proyecciones

Vol. 17, $\mathrm{N}^{\circ} 1$, pp. 23-54, July 1998

Universidad Católica del Norte

Antofagasta - Chile

\title{
COMPENSATORS FOR SINGULAR CONTROL SYSTEMS WITH SMALL DELAYS IN OUTPUTS
}

\author{
HERNÁN R. HENRIQQUEZ* \\ Universidad de Santiago, Santiago-Chile. \\ GENARO A. CASTILLO \\ Universidad de Talca, Talca-Chile.
}

\begin{abstract}
In this paper we study the design of dynarnic compensators for linear singular control systems described by the equation $E x^{\prime}(t)=$ $A x(t)+B u(t)$ with time delayed observed output $y(t)=C x(t-r)$. The proposed compensators are applied to solve the regulator problem for the mentioned systems with controlled output $z(t)=D x(t)$. We also establish a result of existence of exponentially bounded solutions of the retarded singular differential equation $E x^{\prime}(t)=A x(t)+R x(t-$ $r)+f(t), t>0$, with initial condition $x(\theta)=\varphi(\theta),-r \leq \theta \leq 0$.
\end{abstract}

1991 Mathematics Subject Classification. 93D15, 93B52, $93 B 53$.

Key Words and Phrases. Singular Control Systems, Asymptotic Observators, Asymptotic Compensators, Asymptotic Regulators.

"This work was supported in part by FONDECYT, Project 1970716 and by DICYTUSACH , Project 04-9633HM. 


\section{Introduction}

This paper is devoted to the dynamic compensation problem for finitedimension singular systems.

Consider the linear invariant singular system

$$
E x^{\prime}(t)=A x(t)+B u(t)
$$

where $x(t) \in \boldsymbol{R}^{n}$ denotes the state at time $t$ of the system; $u(t) \in$ $\boldsymbol{R}^{m}$, represent the input at time $t$ and both $E, A \in \boldsymbol{R}^{n \times n}$ as $B \in$ $\boldsymbol{R}^{n \times m}$ are constant matrices. When $E=I$ or, in general, $E$ is an invertible matrix, the system (1.1) is called normal and singular (also called descriptor, semistate or generalized) in the case where $\operatorname{det}(E)=0$.

Henceforth we will be concerned with a system (1.1) of singular type. In order to guarantee existence of solutions of equation (1.1), we will assume that the pencil $(E, A)$ is regular in the following sense: there is $\alpha \in \boldsymbol{R}$ such that $\operatorname{det}(\alpha E-A) \neq 0$.

These systems arise in the study of several control problems in science and technology. By this reason, in recent years it has been an increasing interest to study them. Readers are referred to Dai [7] and Campbell $[4,5]$ as well as the references contained therein for the details.

On the other hand, the problem of stabilizing a linear invariant control system by a dynamic output feedback has a very extense literature. At present the theory for normal control systems of finite dimension is well established and we refer to O'Reilly [32] and Wonham [46] for the most important part of the theory. The extension of these results to singular systems has attracted the attention of many authors last years. In particular, the pole assignment problem and the design of asymptotic observers and compensators for the system (1.1) has been considered in several works $([7,13,16,27,30,34,35,36,39,47])$. This work has been concentrated on systems with observed output given by

$$
y(t)=C x(t)
$$

In these systems the observation is instantaneous. However in most of concrete systems their operation presents some time lag. The purpose of this note is to study the dynamic compensation for singular systems described by the equation (1.1) and having a time delay in the observed output. We will restrict us to consider a point delay in the observer variables. Thus, more specifically, our first objective is to determine a dynamic compensator 
for the system (1.1) with the vector output $y(t) \in \boldsymbol{R}^{p}$ at time $t$ given by

$$
y(t)=C x(t-r),
$$

where $C \in \boldsymbol{R}^{p \times n}$ is a time-invariant matrix and the constant $r>0$ represents the time delay of the observation. After, we will apply this compensator to design a tracking controller for the system (1.1)-(1.2) with an appropriate controlled output.

The theory of existence and uniqueness of solutions for the system (1.1) has been discussed by several authors (see Campbell [4] and Dai [7] and the references given therein). In particular, (1.1)-(1.2) is equivalent to the system defined by

$$
\begin{aligned}
x_{1}^{\prime}(t) & =A_{1} x_{1}(t)+B_{1} u(t), \\
N x_{2}^{\prime}(t) & =x_{2}(t)+B_{2} u(t) \\
y(t) & =C_{1} x_{1}(t-r)+C_{2} x_{2}(t-r)
\end{aligned}
$$

where $x_{1} \in \boldsymbol{R}^{n_{1}}, x_{2} \in \boldsymbol{R}^{n_{2}}, n=n_{1}+n_{2}$ and the matrices $A_{1}, B_{1}, B_{2}, C_{1}$, $C_{2}$ and $N$ have appropriate dimensions (see [7] for the terminology). Furthermore, the matrix $N$ is nilpotent with index $h$. The system (1.3)(1.4)-(1.5) is called standard form of (1.1)-(1.2) and is obtained applying a transformation of coordinates defined by invertible matrices $P$ and $Q$ so that

$$
\begin{array}{rlrl}
x & =P\left[\begin{array}{l}
x_{1} \\
x_{2}
\end{array}\right] ; \quad Q E P=\left[\begin{array}{cc}
I & 0 \\
0 & N
\end{array}\right] ; \quad Q A P=\left[\begin{array}{cc}
A_{1} & 0 \\
0 & I
\end{array}\right] \\
Q B & =\left[\begin{array}{l}
B_{1} \\
B_{2}
\end{array}\right] ; & C P & =\left[C_{1}, C_{2}\right] .
\end{array}
$$

The subsystems (1.3) and (1.4) are called slow and fast subsystem, respectively. Since (1.3) is a normal system, the state $x_{1}(t)$ can be obtained from the variation of constants formula. Thus,

$$
x_{1}(t)=e^{A_{1} t} x_{1}(0)+\int_{0}^{t} e^{A_{1}(t-s)} B_{1} u(s) d s .
$$

Furthermore, if $u(\cdot)$ is sufficiently smooth, the solution of (1.4) is given by

$$
x_{2}(t)=-\sum_{i=0}^{h-1} N^{i} B_{2} u^{(i)}(t) .
$$


This paper is organized as follows. Section 2 is dedicated to the construction of the dynamic compensator. In section 3 we apply this compensator to the problem of tracking a signal with regulation of a constant disturbances and in section 4 we extend the results of section 3 to include some non constant disturbances. Finally, we have included three appendixes with some technical results that are needed in these sections.

\section{Design of dynamic compensator}

In this section we shall be concerned with the design of asymptotic compensators for singular systems with delayed observed output.

The problem of feedback stabilization of normal control systems with delays has been discussed in many works, employing different approaches. In particular, our purposes in this paper are related to the results obtained via the finite spectrum assignment method. Some authors have studied different aspects of the problem of stabilization for a fixed time delay (see $[2,6,15,26,29,31,33,37,40,41,42,43,44,38,20])$ while some others have considered the problem of stabilization independent of delays ( $[21$, $28,3,11,22,23,10,12,8,45,25,14,6])$. On the other hand, some of these works are concentrated on the design of asymptotic observers with point delays (commensurate or noncommensurate) while some others consider distributed delays.

In this work we extend the approach of $[26,41,20]$ to construct a dynamic compensator for a singular system with delayed observed output. We consider only a point delay and the proposed compensator will be a normal system with the same point delay. The principal restriction to achieve our objective will be that the time delay $r$ must to be small enough, in a sense that we will explain later.

We begin by designing a singular observer for the system (1.1)-(1.2). To this end we consider the concept of solution introduced in appendix $\mathrm{A}$. Consequently, we assume that the initial condition $x(\theta),-r \leq \theta \leq 0$, is of exponential type, in the sense considered in the Theorem A.1.

We will say that the retarded singular system

$$
E z^{\prime}(t)=A z(t)+G C z(t-r)-G y(t)+B u(t),
$$

is a state observer of (1.1)-(1.2) if

$$
\lim _{t \rightarrow \infty}(z(t)-x(t))=0
$$


Theorem 2.1. Suppose that there exist a matrix $G_{1}$ such that the syst

$$
w^{\prime}(t)=A_{1} w(t)+G_{1} C_{1} w(t-r)
$$

is asymptotically stable. Then there exists a matrix $G$ such that th system (2.1) is a state observer of (1.1)-(1.2).

Proof. Let $e(t):=z(t)-x(t)$ be the estimation of the error. Then

$$
G e^{\prime}(t)=\Lambda e(t)+G C \cdot(t-r) .
$$

Using the coordinate transformation to reduce the pencil $(E, A)$ to the standard form, we obtain that (2.3) is equivalent to the equations

$$
\begin{aligned}
e_{1}^{\prime}(t) & =A_{1} e_{1}(t)+Q_{1} G C P e(t-r), \\
N e_{2}^{\prime}(t) & =e_{2}(t)+Q_{2} G C P e(t-r) .
\end{aligned}
$$

We can choose $G$ so that $Q G$ has the block form $Q G=\left[\begin{array}{c}G_{1} \\ 0\end{array}\right]$.

Since $C P=\left\lfloor C_{1}, C_{2}\right\rfloor$, substituting these expressions into (2.4) and (2.5) we obtain

$$
\begin{aligned}
c_{1}^{\prime}(t) & =A_{1} e_{1}(t)+G_{1} C_{1} c_{1}(t-r)+C_{1}\left(c_{2} c_{2}(t-r),\right. \\
N e_{2}^{\prime}(t) & =c_{2}(t) .
\end{aligned}
$$

From the last equation we infer that $e_{2}(t)=0$, for every $t>0$, which in tiurn implies that

$$
e_{1}^{\prime}(t)=A_{1} e_{1}(t)+G_{1} C_{1} c_{1}(t-r), \quad t>r,
$$

which completes the proof.

Next we will use repeatedly the following result of Halanay (|17], section 4.5). Assuming that the system

$$
x^{\prime}(t)=(A+B) x(t)
$$

is asymptotically stable then there are strictly positive constants $M, \alpha$ such that

$$
\|x(l)\| \leq M c^{-r \ell t}\|x(0)\|, \quad l \geq 0
$$

for all initial condition $x(0)$. Then the delay system

$$
x^{\prime}(l)=A x(l)+B x(t-r)
$$


also is asymptotically stable for

$$
0 \leq r \leq \frac{\alpha}{M\|B\|(\|A\|+\|B\|)} .
$$

In particular, if a pair $(A, B)$ is stabilizable then there exists $F$ such that

$$
x^{\prime}(t)=(A+B F) x(t)
$$

is stable. Consequently, we can associate to $(A, B)$ the constant

$$
\gamma(A, B):=\frac{\alpha}{M\|B F\|(\|A\|+\|B F\|)}
$$

so that for all $0 \leq r \leq \gamma(A, B)$ we have that the system

$$
x^{\prime}(t)=A x(t)+B u(t-r)
$$

is stabilizable with the feedback control $u=F x$. It is clear that similar statements hold for a detectable pair $(A, C)$ and that the following result follows from these observations.

Corollary 2.1. If $\left(A_{1}, C_{1}\right)$ is detectable and $r \leq \gamma\left(A_{1}, C_{1}\right)$, then there exists a matrix $G$ such that the system (2.1) is a state observer of (1.1)(1.2).

The observer proposed in (2.1) is a system of singular type with delays in the state. Thus, this approach is simple but has the disadvantage that the solutions are not continuous functions. To avoid this bad behavior, next we will show that, under appropriated hypotheses, we can define observers of normal type. In fact, following Uetake [39] we can transform the system (1.1) in the form

$$
\widetilde{E}\left[\frac{d x}{d t}-\mu x(t)\right]=x(t)+\widetilde{B} u(t)
$$

where $\widetilde{B}$ and $\widetilde{E}$ are defined by

$$
\widetilde{E}:=(A-\mu E)^{-1} E, \quad \widetilde{B}:=(A-\mu E)^{-1} B
$$

and $\mu$ is a constant such that $\mu E-A$ is an invertible matrix. If the system $(E, A, C)$ is observable then there exists a matrix $G$ such that $L:=\widetilde{E}-G C$ is invertible and the polynomial $\operatorname{det}((s-\mu) L-I)$ is stable (Uetake [39], Theorem 2). Now, we introduce the system

$$
\begin{aligned}
\psi^{\prime}(t) & =L^{-1}(I+\mu L) \psi(t)-L^{-2} G y(t)+L^{-1} \tilde{B} u(t-r) \\
\phi(t) & =\psi(t)-L^{-1} G y(t)
\end{aligned}
$$


and define the error variable $e(t):=\phi(t)-x(t-r)$. Then $(2.9)-(2.10)$ is an asymptotic observer of the system (1.1)-(1.2). In fact, from (2.9)-(2.10) we obtain that

$$
L \phi^{\prime}(t)=(I+\mu L) \phi(t)+\mu G y(t)+\widetilde{B} u(t-r)-G y^{\prime}(t)
$$

which, together with (2.7), implies that

$$
L e^{\prime}(t)=(I+\mu L) e(t) .
$$

Thus

$$
e^{\prime}(t)=L^{-1}(I+\mu L) e(t)
$$

which is a stable system ([39]).

As a first application of the asymptotic observer (2.9)-(2.10) we consider the design of a dynamic compensator for the system (1.1)-(1.2).

We consider the control law

$$
u(t)=F \phi(t) .
$$

Theorem 2.2. Assume that the system $(E, A, C)$ is observable and that the pair $\left(A_{1}, B_{1}\right)$ is controllable. If $0 \leq r \leq \gamma\left(A_{1}, B_{1}\right)$, then there are matrices $F \in \mathbb{R}^{n \times m}$ and $G \in \boldsymbol{R}^{n \times p}$ such that the dynamical system (2.9)-(2.10)-(2.13) is an asymptotic compensator of (1.1)-(1.2).

Proof. Using the controllability condition of $\left(A_{1}, B_{1}\right)$ and the perturbation result of Halanay [17] we can assert that there exists a matrix $F_{1}$ for which the system

$$
z^{\prime}(t)=A_{1} z(t)+B_{1} F_{1} z(l-r)
$$

is asymptotically stable. Defining $F$ so that $F P:=\left[F_{1}, 0\right]$ and substituting $u(\cdot)$ given by $(2.13)$ in (1.3)-(1.4) we obtain that

$$
\begin{aligned}
x_{1}^{\prime}(t) & =A_{1} x_{1}(t)+B_{1} F_{1} x_{1}(t-r)+B_{1} F_{1} e_{1}(t), \\
N x_{2}^{\prime}(t) & =x_{2}(t)+B_{2} F_{1} x_{1}(t-r)+B_{2} F_{1} e_{1}(t) .
\end{aligned}
$$

We observe that this system is singular but, not singular with delay because the last equation does not depend on $x_{2}(t-r)$. On the other hand, from the properties of differentiability of solutions of retarded differential equations $([18])$ we know that $x_{1}(\cdot)$ is a function of class $C^{(h-1)}$ for $t>(h-1) r$. Combining the stability properties of solutions of retarded differential equations (see appendix B) with thuse of system (2.12) we conclude that $x_{1}^{(i)}(t)$ 
converges exponentially to zero as $l$ gues to infinity for all $i \in \mathbb{N}_{0}$. Furthermore, the last equation and (1.7) show that, $x_{2}(t)$ converges exponentially to zero as $t \rightarrow \infty$. From this it follows that $x(t)$ also converges exponentially to zero as $t \rightarrow \infty$ which completes the proof.

\section{Tracking and regulation of constant disturbances}

Now we will employ our previous result,s to design a controller which regulates (i.e. remove the dependence on disturbances) and tracks (i. e. gets the control variables to follow a reference signal) the system. Usually, we will ablreviate our terminulogy saying that a controller with these properties is a regulator or that regulates the given system. In this section we only consider constant disturbances. Specifically we consider a control system

$$
\begin{aligned}
F_{x}{ }^{\prime}(t) & =A x(t)+B u(l)+w, \\
y(t) & =(x)(t-r), \\
z(t) & =D_{x}(t)
\end{aligned}
$$

where $z(l) \in \boldsymbol{I}^{q}$ denotes the controlled output, $w$ designates an unknown constant which represents an external disturbance and $D \in \boldsymbol{R}^{q \times n}$ is a matrix. Oır objective is to design a dynamic compensator such that, the resulting closed-loop system with the perturbation $u=0$ will be exponentially stable and the controlled output $z(l)$ will be regnulated to a reference signal $\bar{z}$. Moreover, this property would occur for all $w$ in a given class of perturbations. Consequently, we discuss here this problem in the case $u$ is a constant perturbation.

Proceeding as above, equation (3.1) can be expressed as

$$
\tilde{E}\left[\frac{d x}{d l !}-\mu x(t)\right\rfloor=x(t)+\tilde{B} u(t)+\tilde{w}
$$

where

$$
\widetilde{w}:=(\Lambda-\mu l s)^{-1} w .
$$

Next, we shall show that, under certain conditions, we can use the previous construction, which requires the delay $r$ to be enough small, to define a PI feedback control to regulate the system. To this end, we intrudice a new variable

$$
\left.\xi^{\prime}(t)=I\right) \phi(t)-M \psi^{\prime}(t)-\bar{z}
$$

where $M$ is an appropriate matrix, and define a PI control by

$$
u(t)=\operatorname{lo} \xi(t)+l d(t) .
$$


Let $\phi_{1}$ and $\phi_{2}$ be the components of $\phi$ corresponding to the transformation $\phi:=P\left[\begin{array}{l}\phi_{1} \\ \phi_{2}\end{array}\right]$. We first show that the pair $\left(\xi, \phi_{1}\right)$ can be considered as the state variable of a system with delays in the control variables.

We begin by rewriting the equation for $\xi$. Substituting the control $u(\cdot)$ and $\psi^{\prime}$ given by (3.7) and (2.9), respectively, into (3.6) and using both (2.10) as the definition of $e(t)$ we obtain that $\xi^{\prime}(t)=-M L^{-1} \widetilde{B} F_{0} \xi(t-r)+\left(D-M L^{-1}(I+\mu L)-\mu M L^{-1} G C\right) \phi(t)$

$$
\xi \prime(t)=+\mu M L^{-1} G C e(t)-M L^{-1} \widetilde{B} F \phi(t-r)-\bar{z} .
$$

We can avoid the dependence of the right hand side of $(3.8)$ on $\phi_{2}$ proceeding as follows. If we put

$$
P^{-1} L P:=\left[\begin{array}{ll}
L_{1,1} & L_{1,2} \\
L_{2,1} & L_{2,2}
\end{array}\right]
$$

then we can select the matrix $G$ so that $L_{1,1}$ will be an invertible matrix (see Lemma C.1 in the appendix C). Hence, if $T$ stands for the inverse of $P^{-1} L P$, then we can write

$$
T:=P^{-1} L^{-1} P:=\left[\begin{array}{ll}
T_{1,1} & T_{1,2} \\
T_{2,1} & T_{2,2}
\end{array}\right]
$$

where the block $T_{2,2}$ is also invertible. Since

$$
\begin{aligned}
I+\mu L+\mu G C & =I+\mu \widetilde{E} \\
& =(A-\mu E)^{1} A
\end{aligned}
$$

$$
\begin{aligned}
\text { then } & \left.\begin{array}{rl}
D P-M L^{-1}(I+\mu L+\mu G C) P & =D P-M L^{-1}(A-\mu E){ }^{1} A P \\
& =D P-M P T(Q(A-\mu E) P)^{1} Q A P \\
& =\left[D_{1}, D_{2}\right] \\
& -\left[M_{1}, M_{2}\right] T\left[\begin{array}{c}
\left(A_{1}-\mu I\right)^{-1} A_{1} \\
0
\end{array}\right. \\
0 \\
0 \\
(I-\mu N)^{-1}
\end{array}\right] \\
& :=\left[\Omega_{1}, \Omega_{2}\right],
\end{aligned}
$$


where we have introduced the notations $D P:=\left[D_{1}, D_{2}\right], M P:=\left[M_{1}, M_{2}\right]$ and

$$
\begin{aligned}
& \Omega_{1}:=D_{1}-M_{1} T_{1,1}\left(A_{1}-\mu I\right)^{-1} A_{1}-M_{2} T_{2,1}\left(A_{1}-\mu I\right)^{-1} A_{1} \\
& \Omega_{2}:=D_{2}-M_{1} T_{1,2}(I-\mu N)^{-1}-M_{2} T_{2,2}(I-\mu N)^{-1} .
\end{aligned}
$$

As $T_{2,2}$ is invertible we can choose $M_{2}$ so that $\Omega_{2}=0$. Consequently, henceforth we will assume that

$$
D_{2}-M_{1} T_{1,2}(I-\mu N)^{-1}-M_{2} T_{2,2}(I-\mu N)^{-1}=0 .
$$

Selecting now a matrix $F$ so that $F P:=\left[F_{1}, 0\right]$ and substituting these expressions into (3.8) we have

$$
\xi^{\prime}(t)=\Omega_{1} \phi_{1}(t)+\mu\left[M_{1}, M_{2}\right] P^{-1} L^{-1} G C e(t)
$$

$$
-\left[M_{1}, M_{2}\right\rfloor P^{-1} L^{-1} \widetilde{B}\left(F_{0} \xi(t-r)+F_{1} \phi_{1}(t-r)\right)-\bar{z} .
$$

On the other hand, proceeding in similar way as above, $(2.11)$ becomes

$$
\begin{aligned}
\phi^{\prime}(t)= & L^{-1}(I+\mu L+\mu G C) \phi(t)-L^{-1} G C \phi^{\prime}(t)+L^{-1} G C\left(e^{\prime}(t)-\right. \\
& -\mu e(t))+L^{-1} \tilde{B}\left(F_{0} \xi(t-r)+F_{1} \phi_{1}(t-r)\right)
\end{aligned}
$$

so that

which implies that

$$
\begin{aligned}
(L+G C) \phi^{\prime}(t)= & (I+\mu L+\mu G C) \phi(t)+G C\left(e^{\prime}(t)-\mu e(t)\right)+\widetilde{B}\left(F_{0} \xi(t-r)+\right. \\
& \left.+F_{1} \phi_{1}(t-r)\right)
\end{aligned}
$$

$E \phi^{\prime}(t)=A \phi(t)+(A-\mu E) G C\left(e^{\prime}(t)-\mu e(t)\right)+B\left(F_{0} \xi(t-r)+F_{1} \phi_{1}(t-r)\right)$.

Applying the transformation of coordinates defined by $P$ and $Q$, the above expression yields

$$
\begin{aligned}
\phi_{1}^{\prime}(t)= & A_{1} \phi_{1}(t)+\left[A_{1}-\mu I, 0\right] P^{-1} G C\left(e^{\prime}(t)-\mu e(t)\right) \\
& +B_{1}\left(F_{0} \xi(t-r)+F_{1} \phi_{1}(t-r)\right)
\end{aligned}
$$

and

$$
\begin{aligned}
N \phi_{2}^{\prime}(t)= & \phi_{2}(t)+[0, I-\mu N] P^{-1} G C\left(e^{\prime}(t)-\mu e(t)\right) \\
& +B_{2}\left(F_{0} \xi(t-r)+F_{1} \phi_{1}(t-r)\right) .
\end{aligned}
$$


Employing the same argument already used in the proof of Theorem 2.2 we can point out that (3.11)-(3.12) is a singular system but not a retarded singular system. It is clear that equations (3.10) and (3.11) can be rewritten as

$$
\frac{d}{d t}\left[\begin{array}{c}
\xi(t) \\
\phi_{1}(t)
\end{array}\right]=\left[\begin{array}{cc}
0 & \Omega_{1} \\
0 & A_{1}
\end{array}\right]\left[\begin{array}{c}
\xi(t) \\
\phi_{1}(t)
\end{array}\right]+\left[\begin{array}{c}
-M L^{-1} \widetilde{B} \\
B_{1}
\end{array}\right]\left[F_{0}, F_{1}\right]\left[\begin{array}{c}
\xi(t-r) \\
\phi_{1}(t-r)
\end{array}\right]
$$

where

$$
f(t):=\left[\begin{array}{c}
\mu M L^{-1} G C e(t)-\bar{z} \\
{\left[A_{1}-\mu I, 0\right] P^{-1} G C\left(e^{\prime}(t)-\mu e(t)\right)}
\end{array}\right] .
$$

In addition, it is easy to see that the estimation of the error $e(t)$ satisfies

$$
e^{\prime}(t)=L^{-1}(I+\mu L) e(t)-L^{-1} \widetilde{\omega} .
$$

In order to study the stability of system (3.13) we introduce the following control system, with delayed control action,

$$
\frac{d}{d t}\left[\begin{array}{c}
\xi(t) \\
\phi_{1}(t)
\end{array}\right]=\left[\begin{array}{cc}
0 & \Omega_{1} \\
0 & A_{1}
\end{array}\right]\left[\begin{array}{c}
\xi(t) \\
\phi_{1}(t)
\end{array}\right]+\left[\begin{array}{c}
-M L^{-1} \widetilde{B} \\
B_{1}
\end{array}\right] v(t-r),
$$

with state space $\boldsymbol{R}^{q} \times \boldsymbol{R}^{n_{1}}$ and control space $\boldsymbol{R}^{m}$. Next we denote by $S$ the $\left(q+n_{1}\right) \times\left(m+n_{1}\right)$ matrix

$$
S:=\left[\begin{array}{cc}
D_{1} & D_{2} B_{2} \\
-A_{1} & B_{1}
\end{array}\right] .
$$

Lemma 3.1. If the pair $\left(A_{1}, B_{1}\right)$ is controllable and the rank of $S$ is $q+n_{1}$, then the system

$$
\frac{d}{d t}\left[\begin{array}{c}
\xi(t) \\
\phi_{1}(t)
\end{array}\right]=\left[\begin{array}{cc}
0 & \Omega_{1} \\
0 & A_{1}
\end{array}\right]\left[\begin{array}{c}
\xi(t) \\
\phi_{1}(t)
\end{array}\right]+\left[\begin{array}{c}
-M L^{-1} \widetilde{B} \\
B_{1}
\end{array}\right] v(t)
$$

also is controllable.

Proof. The assertion is consequence of the Hautus test ([19]). In fact, if we use $\rho$ to indicate the rank of a matrix and since $\rho\left[\lambda I-A_{1}, B_{1}\right]=n_{1}$, for every $\lambda \in \mathscr{C}$, then

$$
\begin{aligned}
\rho\left[\lambda I-\left[\begin{array}{cc}
0 & \Omega_{1} \\
0 & A_{1}
\end{array}\right],\left[\begin{array}{c}
-M L^{-1} \widetilde{B} \\
B_{1}
\end{array}\right]\right] & =\rho\left[\begin{array}{ccc}
\lambda I & -\Omega_{1} & -M L^{-1} \widetilde{B} \\
0 & \lambda I-A_{1} & B_{1}
\end{array}\right] \\
& =q+n_{1},
\end{aligned}
$$


for all $\lambda \neq 0$. Moreover, in order to estimate the rank in the case $\lambda=0$ we begin by observing that

$$
\begin{aligned}
M L^{-1} \widetilde{B}= & {\left[M_{1}, M_{2}\right] T P^{-1} \widetilde{B} } \\
= & {\left[M_{1}, M_{2}\right] T P^{-1}(A-\mu E)^{-1} B } \\
= & {\left[M_{1}, M_{2}\right] T(Q(A-\mu E) P)^{-1} Q B } \\
= & M_{1}\left(T_{1,1}\left(A_{1}-\mu I\right)^{-1} B_{1}+T_{1,2}(I-\mu N)^{-1} B_{2}\right) \\
& +M_{2}\left(T_{2,1}\left(A_{1}-\mu I\right)^{-1} B_{1}+T_{2,2}(I-\mu N)^{-1} B_{2}\right) .
\end{aligned}
$$

This expression and the definition of $\Omega_{1}$ allow us to write

$$
\begin{aligned}
& {\left[\begin{array}{cc}
\Omega_{1} & M L^{-1} \widetilde{B} \\
-A_{1} & B_{1}
\end{array}\right] } \\
= & {\left[\begin{array}{cc}
I & \left(M_{1} T_{1,1}+M_{2} T_{2,1}\right)\left(A_{1}-\mu I\right)^{-1} \\
0 & I \\
D_{1} & \left(M_{1} T_{1,2}+M_{2} T_{2,2}\right)(I-\mu N)^{-1} B_{2} \\
-A_{1} & B_{1}
\end{array}\right] }
\end{aligned}
$$

and combining this equality with (3.9) and our hypothesis about the rank of $S$ it follows that

$$
\begin{aligned}
\rho\left[\begin{array}{cc}
\Omega_{1} & M L^{-1} \tilde{B} \\
-A_{1} & B_{1}
\end{array}\right] & =\rho\left[\begin{array}{cc}
D_{1} & \left(M_{1} T_{1,2}+M_{2} T_{2,2}\right)(I-\mu N)^{-1} B_{2} \\
-A_{1} & B_{1}
\end{array}\right] \\
& =\rho\left[\begin{array}{cc}
D_{1} & D_{2} B_{2} \\
-A_{1} & B_{1}
\end{array}\right] \\
& =q+n_{1} .
\end{aligned}
$$

Next we denote by $\gamma$ the constant associated to system (3.17) and defined by $(2.6)$.

We are now in condition to prove the following result.

Proposition 3.1. Assume that $(E, A, C)$ is observable and $\left(A_{1}, B_{1}\right)$ is controllable. If the rank of $S$ is $q+n_{1}$ and $r \leq \gamma$, then there are matrices $F_{0}, F_{1}$ and $G$ such that the system (2.9)-(2.10)-(3.6) with the control law (3.7) is asymptotically stable and $\lim _{t \rightarrow \infty} D \phi(t)=\bar{z}$. If we assume 
further that the disturbance $w=0$ then the system (2.9)-(2.10)-(3.6) with the control law (3.7) regulates the control system (3.1)-(3.2)-(3.3).

Proof. Using the already mentioned perturbation result of Halanay and the previous lemma we conclude the existence of a pair of matrices $F_{0}$ and $F_{1}$ so that the feedback control law

$$
v(t)=F_{0} \xi(t)+F_{1} \phi_{1}(t)
$$

stabilizes the system (3.15).

Since $f(t)$ is convergent as $t \rightarrow \infty$, applying Proposition B.1 to systems (3.14) and (3.13) we obtain that the functions $e(t), \xi(t)$ and $\phi_{1}(t)$ have limit at $\infty$. Similarly, from (3.12) it follows that $\phi_{2}(t)$ also converges as $t \rightarrow \infty$. Next we represent by $\bar{e}, \bar{\xi}, \bar{\phi}_{1}$ and $\bar{\phi}_{2}$ the limit at infinity of $e(t), \xi(t), \phi_{1}(t)$ and $\phi_{2}(t)$, respectively. Consequently, from (3.14) we obtain that

$$
\bar{e}=(I+\mu L)^{-1} \widetilde{w}
$$

and, substituting this value in $(3.10),(3.11)$ and (3.12) we obtain the following set of equations

$$
\begin{aligned}
& \begin{array}{l}
\Omega_{1} \bar{\phi}_{1}+\mu\left[M_{1}, M_{2}\right] P^{-1} L^{-1} G C(I+\mu L)^{-1} \widetilde{w} \\
-\left[M_{1}, M_{2}\right] P^{-1} L^{-1} \widetilde{B}\left(F_{0} \bar{\xi}+F_{1} \bar{\phi}_{1}\right)-\bar{z}=0
\end{array} \\
& A_{1} \bar{\phi}_{1}-\mu\left[A_{1}-\mu I, 0\right] P^{-1} G C(I+\mu L)^{-1} \widetilde{w}+B_{1}\left(F_{0} \bar{\xi}+F_{1} \bar{\phi}_{1}\right)=0
\end{aligned}
$$

$$
\bar{\phi}_{2}-\mu[0, I-\mu N] P^{-1} G C(I+\mu L)^{-1} \tilde{w}+B_{2}\left(F_{0} \bar{\xi}+F_{1} \bar{\phi}_{1}\right)=0
$$

Since (3.19) and (3.20) are equivalent to

$$
A \bar{\phi}-\mu(A-\mu E) G C(I+\mu L)^{-1} \widetilde{w}+B\left(F_{0} \bar{\xi}+F_{1} \bar{\phi}_{1}\right)=0
$$

multiplying this expression by $\left[M_{1}, M_{2} \mid P^{-1} L^{-1}(A-\mu E)^{-1}\right.$ it yields that 


$$
\begin{gathered}
\left\lfloor M_{1}, M_{2} \mid P^{-1} L^{-1}(A-\mu E)^{-1} A \bar{\phi}=\mu\left[M_{1}, M_{2}\right\rfloor P^{-1} L^{-1} G C(I+\mu L)^{-1} \widetilde{w}-\right. \\
-\left\lfloor M_{1}, M_{2}\right\rfloor P^{-1} L^{-1} \widetilde{B}\left(F_{0} \bar{\xi}+\right. \\
\left.+F_{1} \bar{\phi}_{1}\right) .
\end{gathered}
$$

Substituting the right hand side of the above expression in (??) we see that,

$$
\Omega_{1} \bar{\phi}_{1}+\left\lfloor M_{1}, M_{2} \mid P^{-1} L^{-1}(A-\mu E)^{-1} A \bar{\phi}-\bar{z}=0\right.
$$

which, by the definition of $\Omega_{i}, i=i, 2$, implies that $D \bar{\phi}=\bar{z}$. Finally, since $\bar{e}=\bar{\phi}-\bar{x}$ and $\bar{e}=(I+\mu L)^{-1} \tilde{u}$, we conclude that $D \bar{x}=$ $\bar{z}-(I+\mu L)^{-1} \widetilde{w}$, which completes the proof.

\section{Tracking and regulation of some nonconstant disturbances}

The conclusion of section 3 also remains valid for some non zero, yet non constant, disturbances. To obtain this extension we consider the regulator problem for the singnlar system

$$
\begin{aligned}
W_{x}^{\prime}(t) & =A x(t)+B u(t)+W u(t), \\
y(t) & =C x(t-r) \\
z(t) & =D x(t)
\end{aligned}
$$

where the disturbance $w(t) \in \mathbb{R}^{d}$ satisfies the equation

$$
w^{\prime}(t)=V w(t)
$$

for some matrices $W$ and $V$ of appropriate dimensions.

Proceeding as in Dai ([7]) we can treat the regulator problem for this system as the regulator problem for a system without perturbations. In fact, using the definition $x_{a}:=\left[\begin{array}{c}x \\ u\end{array}\right]$ we represent (4.1-4.4) as the augmented syst.em

$$
\begin{aligned}
E_{a} x_{a}^{\prime}(t) & =A_{a} x_{a}(t)+B_{a} u(t), \\
y(t) & =C_{a}^{\prime} x_{a}(t-r), \\
z(t) & =D_{a} x_{a}(t)
\end{aligned}
$$

where we have introduced the notations

$$
\begin{array}{ll}
E_{a}:=\left[\begin{array}{cc}
E & 0 \\
0 & I
\end{array}\right] ; & A_{a}:=\left[\begin{array}{cc}
\Lambda & W \\
0 & V
\end{array}\right] ; B_{a}:=\left[\begin{array}{c}
B \\
0
\end{array}\right] ; \\
C_{a}:=\lfloor C, 0] ; & D_{a}:=\lfloor D, 0] .
\end{array}
$$


It is clear that $\left(E_{a}, A_{a}\right)$ is a regular pair so that we can try to apply our preceding construction to solve the regulator problem for the system (4.5)-(4.6)-(4.7). In order to follow the scheme established in section 3 next we will assume that $P, Q, A_{1}, N, B_{1}$ and $B_{2}$ represent the matrices previously defined in connection with the standard form for $(E, A)$.

Proceeding in similar way as in section 2 we define $\widetilde{E_{a}}:=\left(A_{a}-\right.$ $\left.\mu E_{a}\right)^{-1} E_{a}$ and $\widetilde{B_{a}}:=\left(A_{a}-\mu E_{a}\right)^{-1} B_{a}$. If we assume that the system $\left(E_{a}, A_{a}, C_{a}\right)$ is observable then there exists a matrix $G_{a}$ such that $L_{a}:=E_{a}-G_{a} C_{a}$ is invertible and the polynomial $\operatorname{det}\left((s-\mu) L_{a}-I\right)$ is stable. Furthermore, the system

(4.8) $\psi_{a}^{\prime}(t)=L_{a}^{-1}\left(I+\mu L_{a}\right) \psi_{a}(t)-L_{a}^{-2} G_{a} y(t)+L_{a}^{-1} \widetilde{B_{a}} u(t-r)$,

(4.9) $\phi_{a}(t)=\psi_{a}(t)-L_{a}^{-1} G_{a} y(t)$

is an asymptotic observator of (4.5)-(4.6). Specifically, if

$$
e_{a}(t):=\phi_{a}(t)-x_{a}(t-r)
$$

stands for the estimation of the error then

$$
e_{a}^{\prime}(t)=L_{a}^{-1}\left(I+\mu L_{a}\right) e_{a}(t),
$$

which is a stable system.

Next we reduce the system (4.5)-(4.6)-(4.7) to its standard form by repeated transformation of coordinates. In the first term we apply the transformation which is performed by multiplying the equation (4.5) on the left by the matrix $Q_{a}^{1}:=\left[\begin{array}{cc}Q & 0 \\ 0 & I\end{array}\right]$ and by substituting $x_{a}$ by $\left[\begin{array}{ll}P & 0 \\ 0 & I\end{array}\right]\left[\begin{array}{c}x_{1} \\ x_{2} \\ w\end{array}\right]$. Now we apply a transformation of coordinates in such way that the second and third components of vector $\left(x_{1}, x_{2}, w\right)^{T}$ are permuted. It is clear that relative to the new coordinates the equation (4.5) take the form

$$
\begin{aligned}
x_{1}^{\prime}(t) & =A_{1} x_{1}(t)+W_{1} w(t)+B_{1} u(t), \\
w^{\prime}(t) & =V w(t) \\
N x_{2}^{\prime}(t) & =x_{2}(t)+W_{2} w(t)+B_{2} u(t)
\end{aligned}
$$

where $\left[\begin{array}{l}W_{1} \\ W_{2}\end{array}\right]=Q W$. However, this singular system is not yet in standard form. By this reason we proceed to perform a new transformation 
of coordinates which is constructed as in Lemma C.2 of the appendix $C$. To apply this result we put $H:=\left[\begin{array}{cc}A_{1} & W_{1} \\ 0 & V\end{array}\right]$ and $R:=\left[0, W_{2}\right]$ an! define the matrix $X$ by means of (C.3). This new transformation of coordinates is obtained by multiplying the system $(4.124 .14)$ on the left by $Q_{a}^{2}:=\left[\begin{array}{cc}I & 0 \\ -N X & I\end{array}\right]$ and substituting the vector $\left(x_{1}, w, x_{2}\right)^{T}$ according to

$$
\left[\begin{array}{l}
x_{1} \\
w \\
x_{2}
\end{array}\right]:-\left[\begin{array}{ll}
I & 0 \\
X & I
\end{array}\right]\left[\begin{array}{c}
x_{1} \\
w \\
x_{2}
\end{array}\right]
$$

where $\overline{x_{2}}$ is a new variable. 'The system obtained by applying to (4.12)(4.13)-(4.14) this transformation is given by

$$
\begin{aligned}
& x_{1}^{\prime}(t)=A_{1} x_{1}(t)+W_{1} w(t)+\overline{B_{1}}(i) \\
& w^{\prime}(t)=V w(t) \\
& N \overline{x_{2}^{\prime}}(t)=\overline{x_{2}}(t)+\overline{B_{2}} u(t) \\
& \text { where }\left[\begin{array}{c}
\overline{B_{1}} \\
0 \\
\overline{B_{2}}
\end{array}\right]=\left[\begin{array}{cc}
I & 0 \\
-N X & I
\end{array}\right]\left[\begin{array}{c}
B_{1} \\
0 \\
B_{2}
\end{array}\right] \text {. Furthermore, since } \\
& X=-\sum_{i=0}^{h-1} N^{i}\left[0, W_{2}\right]\left[\begin{array}{cc}
A_{1} & W_{1} \\
0 & V
\end{array}\right]^{i}
\end{aligned}
$$

then

$$
X\left[\begin{array}{c}
B_{1} \\
0
\end{array}\right]=-\sum_{i=0}^{h-1} N^{i}\left[0, W_{2}\right]\left[\begin{array}{cc}
A_{1} & W_{1} \\
0 & V
\end{array}\right]^{i}\left[\begin{array}{c}
B_{1} \\
0
\end{array}\right]=0
$$

which implies that $\overline{B_{1}}=B_{1}$ and $\overline{B_{2}}=B_{2}$.

We denote $Q_{a}:=Q_{a}^{2} Q_{a}^{1}$ and define $P_{a}$ as the product of those transformations of this sequence that take $x_{a}$ into $\left(x_{1}, w, \overline{x_{2}}\right)^{T}$. It is clear that the transformation defined by $Q_{a}$ and $P_{a}$ transfer the system (4.5) into its standard form.

Now we introduce the variable

$$
\xi^{\prime}(t)=D_{a} \phi_{a}(t)-M_{a} \psi_{a}^{\prime}(t)-\bar{z}
$$


and define the control law

$$
u(t)=F_{0} \xi(t)+F_{a} \phi_{a}(t) .
$$

Using the transformation $P_{a}$ we set $\phi_{a}:=P_{a}\left[\begin{array}{c}\phi_{11} \\ \phi_{12} \\ \phi_{2}\end{array}\right]$. Initially, we proceed as in section 3 to show that $\left(\xi, \phi_{11}\right)$ is the variable of a system of type (3.15). We begin by studying the equation for $\phi_{11}$. Proceeding as in the remarks previous to Proposition 3.1, it follows that

$E_{a} \phi_{a}^{\prime}(t)=A_{a} \phi_{a}(t)+\left(A_{a}-\mu E_{a}\right) G_{a} C_{a}\left(e_{a}^{\prime}(t)-\mu e_{a}(t)\right)+B_{a}\left(F_{0} \xi(t-r)+F_{a} \phi_{a}(t-r)\right)$. (4.17)

Selecting $F_{a}$ so that $F_{a} P_{a}=\left[F_{11}, F_{12}, 0\right]$ and multiplying the equation (4.17) on the left by the transformation $Q_{a}$ it is easy to see that the system (4.17) is changed into

$$
\begin{aligned}
\phi_{11}^{\prime}(t)= & A_{1} \phi_{11}(t)+W_{1} \phi_{12}(t)+B_{1}\left[F_{0} \xi(t-r)+F_{11} \phi_{11}(t-r)\right. \\
& \left.+F_{12} \phi_{12}(t-r)\right]+f_{11}(t),
\end{aligned}
$$

$$
\phi_{12} l(t)=V \phi_{12}(t)+f_{12}(t),
$$

$$
\begin{gathered}
\phi_{2}^{\prime}(t)=\phi_{2}(t)+B_{2}\left[F_{0} \xi(t-r)+F_{11} \phi_{11}(t-r)+F_{12} \phi_{12}(t-r)\right]+ \\
+f_{2}(t)
\end{gathered}
$$

where $f_{11}(t), f_{12}(t)$ and $f_{2}(t)$ are certain continuous functions which vanish at infinity.

On the other hand, from (4.19) it follows that

$$
\begin{aligned}
\phi_{12}(t) & =e^{V r} \phi_{12}(t-r)+\int_{t-r}^{t} e^{V(t-s)} f_{12}(s) d s \\
& =e^{V r} \phi_{12}(t-r)+g_{12}(t)
\end{aligned}
$$

where $g_{12}(t) \rightarrow 0$ as $t \rightarrow \infty$. Substituting this expression in (??) we infer that

$$
\begin{aligned}
\phi_{11}^{\prime}(t)= & A_{1} \phi_{11}(t)+\left(W_{1} e^{V r}+B_{1} F_{12}\right) \phi_{12}(t-r) \\
& +B_{1}\left[F_{0} \xi(t-r)+F_{11} \phi_{11}(t-r)\right]+g_{11}(t)
\end{aligned}
$$

for some function $g_{11}$ such that $g_{11}(t) \rightarrow 0$ as $t \rightarrow \infty$.

The above considerations suggest to introduce the additional hypothesis

$$
\rho[B, W]=\rho[B] .
$$


Under this condition we can choose $F_{12}$ so that

$$
W e^{V \tau}+B F_{12}=0
$$

and substituting this in the above expression for $\phi_{11}$ we obtain

$(4.23) \phi_{11}^{\prime}(t)=A_{1} \phi_{11}(t)+B_{1}\left[F_{0} \xi(t-r)+F_{11} \phi_{11}(t-r)\right]+g_{11}(t)$.

Next we derive the equation for $\xi$. From (4.15) we can write

$$
\begin{aligned}
\xi^{\prime}(t) & =-M_{a} L_{a}^{-1} \widetilde{B_{a}} F_{0} \xi(t-r)+\left[D_{a}-M_{a} L_{a}^{-1}\left(I+\mu L_{a}+\mu G_{a} C_{a}\right)\right] \phi_{a}(t) \\
& +\mu M_{a} L_{a}^{-1} G_{a} C_{a} e_{a}(t)-M_{a} L_{a}^{-1} \widetilde{B_{a}} F_{a} \phi_{a}(t-r)-\bar{z}
\end{aligned}
$$

This expression can be modified by replacing

$$
I+\mu L_{a}+\mu G_{a} C_{a}=\left(A_{a}-\mu E_{a}\right)^{-1} A_{a}
$$

and by observing that

$$
D_{a} \phi_{a}(t)=D_{a} P_{a}\left[\begin{array}{c}
\phi_{11}(t) \\
\phi_{12}(t) \\
\phi_{2}(t)
\end{array}\right]=\left[D_{1}, D_{12}, D_{2}\right]\left[\begin{array}{c}
\phi_{11}(t) \\
\phi_{12}(t) \\
\phi_{2}(t)
\end{array}\right]
$$

where $D_{12}:=-\sum_{i=0}^{h-1} D_{2} N^{i} W_{2} V^{i} . \quad$ In fact, from the definition of $P_{a}$ we know that

$$
D_{a} P_{a}=\left[D_{1}, 0, D_{2}\right]\left[\begin{array}{cc}
I & 0 \\
X & I
\end{array}\right]=\left[\left[D_{1}, 0\right]+D_{2} X, D_{2}\right]
$$

and since

$$
\begin{aligned}
D_{2} X & =-\sum_{i=0}^{h-1} D_{2} N^{i}\left[0, W_{2}\right]\left[\begin{array}{cc}
A_{1} & W_{1} \\
0 & V
\end{array}\right]^{i} \\
& =\left[0,-\sum_{i=0}^{h-1} D_{2} N^{i} W_{2} V^{i}\right]
\end{aligned}
$$


the assertion follows. Thus, we find that

$$
\begin{aligned}
& \xi^{\prime}(t)=-M_{a} L_{a}^{-1} \widetilde{B_{a}} F_{0} \xi(t-r)+D_{a} P_{a}\left[\begin{array}{c}
c: 1 i c) \\
\phi_{12}(t) \\
t_{1}(i)
\end{array}\right]+\mu M_{a} L_{a}^{-1} G_{a} C_{a} e_{a}(t)-
\end{aligned}
$$

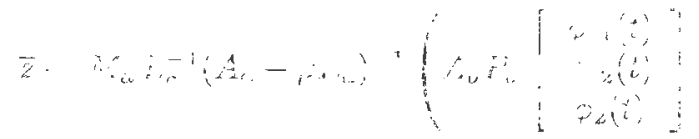

$$
\begin{aligned}
& \left.\left.H_{q_{i}} F_{11}, F_{12}, G\right)\left(\begin{array}{c}
r(2-\cdots) \\
\phi_{12}(t-r) \\
\phi_{2}(t-r)
\end{array}\right]\right) \\
& =-M_{a} \bar{L}_{a}^{-1} \widetilde{B_{a}} F_{0} \xi(t-r)+D_{1} \phi_{11}(t)+D_{12} \phi_{12}(t)+D_{2} \phi_{2}(t) \\
& \left.-i M_{11}^{\prime}, M_{12}^{\prime}, M_{2}^{\prime}\right]\left[\begin{array}{r}
A_{1} \phi_{11}(t)+W_{1} \phi_{12}(t)+B_{1}\left(F_{11} \phi_{11}(t-r)+\right. \\
\left.+F_{12} \phi_{12}(t-r)\right) V \phi_{12}(t) \\
\phi_{2}(t)+B_{2}\left(F_{11} \phi_{11}(t-r)+F_{12} \phi_{12}(t-r)\right)
\end{array}\right] \\
& +\mu M_{a} L_{a}^{-1} G_{a} C_{a} e_{a}(t)-\bar{z}
\end{aligned}
$$

where we have used the notation

$$
M_{a} P_{a}\left(Q_{a}\left(A_{a}-\mu E_{a}\right) L_{a} P_{a}\right)^{-1}:=\left[M_{11}^{\prime}, M_{12}^{\prime}, M_{2}^{\prime}\right] .
$$

Replacing in the last expression for $\xi^{\prime}(t)$ the value of $\phi_{12}(t)$ given by (4.21) and designating $D_{1}-M_{11}^{\prime} A_{1}$ as $\Omega_{1}$ we can write

$$
\begin{aligned}
\xi^{\prime}(t)= & \Omega_{1} \phi_{11}(t)-M_{a} L_{a}^{-1} \widetilde{B_{a}} F_{0} \xi(t-r)-M_{a} L_{a}^{-1} \widetilde{B_{a}} F_{11} \phi_{11}(t-r) \\
& +\left[\left(D_{12}-M_{11}^{\prime} W_{1}-M_{12}^{\prime} V\right) e^{V r}-M_{11}^{\prime} B_{1} F_{12}-\right. \\
& \left.-M_{2}^{\prime} B_{2} F_{12}\right\rfloor \phi_{12}(t-r)+\left(D_{2}-M_{2}^{\prime}\right) \phi_{2}(t)+f(t)-\bar{z}
\end{aligned}
$$

where $f(t)$ is a function that vanishes at $\infty$.

Selecting the matrix $M_{a}$ so that $M_{12}^{\prime}=-D_{2} N \sum_{i=0}^{h-2} N^{i} W_{2} V^{i} ; M_{2}^{\prime}=D_{2}$ and using the condition (4.22) the last expression yields $\xi^{\prime}(t)=\Omega_{1} \phi_{11}(t)-M_{a} L_{a}^{-1} \widetilde{B_{a}} F_{0} \xi(t-r)-M_{a} L_{a}^{-1} \widetilde{B_{a}} F_{11} \phi_{11}(t-r)+f(t)-\bar{z}$ (4.24)

It is clear that, with minor changes in the notations, equations (4.23) and (4.24) can be reformulated as the system (3.13). The matrix $S$ that arises 
in this case is the same already defined in (3.16). Further, proceeding as in the proof of Lemma 3.1 we obtain that the system

$$
\frac{d}{d t}\left[\begin{array}{c}
\xi(t) \\
\phi_{11}(t)
\end{array}\right]=\left[\begin{array}{cc}
0 & \Omega_{1} \\
0 & A_{1}
\end{array}\right]\left[\begin{array}{c}
\xi(t) \\
\phi_{11}(t)
\end{array}\right]+\left[\begin{array}{c}
-M_{a} L_{a}^{-1} \widetilde{B_{a}} \\
B_{1}
\end{array}\right] v(t)
$$

is controllable. In what follows $\gamma$ will stand for the constant associated to this system and defined by (2.6). Taking in consideration that in this formulation the perturbed original system is transformed into the augmented system which is free of disturbances, from Proposition 3.1 we obtain

Theorem 4.1. Assume that the following conditions hold:

(i) The system $\left(E_{a}, A_{a}, C_{a}\right)$ is observable;

(ii) The pair $\left(A_{1}, B_{1}\right)$ is controllable;

(iii) $\rho[B, W]=\rho[B]$;

(iv) $\rho S=q+n_{1}$.

If $0 \leq r \leq \gamma$ then there are matrices $F_{0}, F_{a}$ and $G_{a}$ such that the system (4.8)-(4.9)-(4.15) with the control law (4.16) is an asymptotic regulator of system (4.1)-(4.2)-(4.3)-(4.4).

A well known result of Hayes (see Bellman and Cooke [1]) shows that the preceding results cannot be stated without the condition that the delay $r>0$ is small. However, we may expect to avoid this hypothesis defining a distributed delay control law instead of a proportional state feedback or applying the theory of stabilizability independant of delay. This approach will be the matter of a future work.

\section{A. Appendix}

\section{Existence of solutions of singular differential equations with time delay}

In this appendix we consider the problem of existence of solutions of retarded singular differential equations of type

$$
E x^{\prime}(t)=A x(t)+R x(t-r)+f(t), \quad t>0,
$$

with initial condition

$$
x(\theta)=\varphi(\theta), \quad-r \leq \theta \leq 0,
$$


where $x(t), f(t) \in \mathbb{R}^{n}$ and $E, A, R \in \mathbb{R}^{n \times n}$ are constant matrices.

This problem has been studied in Campbell [4] but this approach only considers infinitely differentiable solutions. In that context the equation (A.1) has a solution only for a restricted set of consistent initial conditions $\varphi$. In studying the design of asymptotic compensators one is led to extend the concept of solution to include non differentiable functions and to enlarge the set of possible initial conditions.

In order to establish our result of existence of solutions for the equation (A.1) we introduce previously some terminology and notations. We will say that a function $x:[0, \infty) \rightarrow \boldsymbol{R}^{n}$ is $r$-piecewise of class $C^{\infty}$ if the restriction of $x(\cdot)$ to each interval $(k r,(k+1) r], k \in \mathbb{N}_{0}$, is a function of class $C^{\infty}$ and there exist the limits of the derivatives $x^{(i)}(t)$, as $t \rightarrow(k r)^{+}$, for all $i, k \in \mathbb{N}_{0}$. We will denote by $C_{r}^{\infty}\left(\boldsymbol{R}^{n}\right)$ the space of $r$-piecewise of class $C^{\infty}$ functions. Moreover, as usual in the theory of functional equations, for a function $x:[-r, \infty) \rightarrow \mathbb{R}^{n}$ we represent by $x_{t}:[-r, 0] \rightarrow \boldsymbol{R}^{n}, t \geq 0$, the function defined by $x_{t}(\theta):=x(t+\theta)$.

Definition A.1. We will say that a function $x:[-r, \infty) \rightarrow \mathbb{R}^{n}$ is a solution of problem (A.1)-(A.2) if $x(\cdot)$ is a r-piecewise of class $C^{\infty}$ function on $[0, \infty)$ that satisfies (A.2) and the equation (A.1) on each interval $(k r,(k+1) r\}, k \in \mathbb{N}_{0}$.

Next we use subindexes 1 and 2 to denote component in the standard form associated to $(E, A)$. In particular, we put $Q R P:=\left[R_{i j}\right]_{i, j=1,2}$. In addition, for a $r$-piecewise of class $C^{\infty}$ function $x(\cdot)$ we denote by $x^{(i)}(t)$ the left derivative of order $i \geq 0$ of $x(\cdot)$ at $t$. Now we are in conditions to establish the following result.

Theorem A.1. Assume that $(E, A)$ is a regular pair. Then for each function $\varphi$ of class $C^{\infty}$ on $[-r, 0]$ and each $f$ of class $C^{\infty}$ on $[0, \infty)$, there exists a unique solution of (A.1)-(A.2). Furthermore, if there exist positive constants $M_{1}, M_{2}, \omega_{1}$ and $\omega_{2}$ such that $\left\|f^{(i)}(t)\right\| \leq M_{1} \omega_{1}^{i} e^{\omega_{1} t}$ and $\left\|\varphi^{(i)}(\theta)\right\| \leq M_{2} \omega_{2}^{i}$, for every $i \in \mathbb{N}_{0}, t \geq 0$ and $-r \leq \theta \leq 0$ then there exist some pasitive constants $M$ and $\omega$ such that $\left\|x^{(i)}(t)\right\| \leq$ $M \omega^{i} e^{\omega t}$ for $i \in \mathbb{N}_{0}$ and every $t>0$.

Proof. The demonstration of the existence is an easy consequence of an inductive argument. In fact, on the interval $(0, r]$ the initial value problem (A.1)-(A.2) is reduced to the equation

$$
E x^{\prime}(t)=A x(t)+R \varphi(t-r)+f(t),
$$


which has a unique solution. Moreover, using the standard decomposition of the pencil $(E, A)$, we obtain that $x(\cdot)$ is a function of class $C^{\infty}$ on $(0, r]$ and that there exist the limits of $x^{(i)}(t)$, as $t \rightarrow 0^{+}$, for every $i \in \mathbb{N}_{\mathbf{0}}$.

If we assume now that $x(\cdot)$ is defined on $[-r, k r]$, for some $k \in \mathbb{N}$, and it is a $r$-piecewise of class $C^{\infty}$ function on this interval, then the same argument already used allow us to extend the solution $x(\cdot)$ to the interval $[-r,(k+1) r]$, with the properties already mentioned.

To prove the second assertion we introduce some notations. First, we consider the spaces of matrices endowed with the operator norm associated to the corresponding vector norm. We set $\alpha_{i j}:=\left\|R_{i j}\right\|$ and $\alpha_{1}:=\left\|A_{1}\right\|$. Certainly we can assume that $\alpha_{1}<\omega_{1}$ and that $\left\|f_{j}^{(i)}(t)\right\| \leq M_{1} \omega_{1}^{i} e^{\omega_{1} t}$ and $\left\|\varphi_{j}^{(i)}(\theta)\right\| \leq M_{2} \omega_{2}^{i}$, for every $j=1,2, \quad i \in \mathbb{N}_{0}, \quad t \geq 0$ and $-r \leq \theta \leq 0$

We will choose the constants $M \geq \max \left\{M_{1}, M_{2}\right\}$ and $\omega \geq \max \left\{\omega_{1}, \omega_{2}\right\}$ so that the following conditions hold:

$$
\begin{aligned}
\sum_{i=0}^{h-1}\|N\|^{i}\left[\left(\alpha_{21}+\alpha_{22}\right) M_{2} \omega_{2}^{i}+M_{1} \omega_{1}^{i}\right] & \leq M, \\
M_{2}\left(1+\frac{\alpha_{11}+\alpha_{12}}{\alpha_{1}}\right)+\frac{M_{1}}{\omega_{1}-\alpha_{1}} & \leq M,
\end{aligned}
$$

$$
\alpha_{1}+\alpha_{11}+\alpha_{12}+1 \leq \omega
$$

As in the first part, we use an inductive argument to prove the assertion. For this reason we begin by proving that the property holds for every $t \in$ $(0, r]$. In fact, since

$$
\begin{aligned}
x_{2}^{(k)}(t) & =-\sum_{i=0}^{h-1} N^{i}\left[R_{21} x_{1}^{(i+k)}(t-r)+R_{22} x_{2}^{(i+k)}(t-r)+f_{2}^{(i+k)}(t)\right] \\
& =-\sum_{i=0}^{h-1} N^{i}\left[R_{21} \varphi_{1}^{(i+k)}(t-r)+R_{22} \varphi_{2}^{(i+k)}(t-r)+f_{2}^{(i+k)}(t)\right]
\end{aligned}
$$


then, from (A.3) we obtain,

$$
\begin{aligned}
\left\|x_{2}^{(k)}(t)\right\| \leq & \sum_{i=0}^{h-1}\|N\|^{i}\left[\alpha_{21}\left\|\varphi_{1}^{(i+k)}(t-r)\right\|+\alpha_{22}\left\|\varphi_{2}^{(i+k)}(t-r)\right\|+\right. \\
& \left.+\left\|f_{2}^{(i+k)}(t)\right\|\right] \\
\leq & \sum_{i=0}^{h-1}\|N\|^{i}\left[M_{2}\left(\alpha_{21}+\alpha_{22}\right) \omega_{2}^{i+k}+M_{1} \omega_{1}^{i+k} e^{\omega_{1} t}\right] \\
\leq & \sum_{i=0}^{h-1}\|N\|^{i}\left[M_{2}\left(\alpha_{21}+\alpha_{22}\right) \omega_{2}^{i}+M_{1} \omega_{1}^{i}\right] \omega^{k} e^{\omega t} \\
\leq & M \omega^{k} e^{\omega t} .
\end{aligned}
$$

On the other hand, we know that

$$
\begin{aligned}
x_{1}(t) & =e^{A_{1} t} x_{1}(0)+\int_{0}^{t} e^{A_{1}(t-s)}\left[R_{11} x_{1}(s-r)+R_{12} x_{2}(s-r)+f_{1}(s)\right] d s \\
& =e^{A_{1} t} \varphi_{1}(0)+\int_{0}^{t} e^{A_{1}(t-s)}\left[R_{11} \varphi_{1}(s-r)+R_{12} \varphi_{2}(s-r)+f_{1}(s)\right] d s
\end{aligned}
$$

therefore

$$
\begin{aligned}
\left\|x_{1}(t)\right\| & \leq M_{2} e^{\alpha_{1} t}+\int_{0}^{t} e^{\alpha_{1}(t-s)}\left[\left(\alpha_{11}+\alpha_{12}\right) M_{2}+M_{1} e^{\omega_{1} s}\right] d s \\
& =M_{2}\left(1+\frac{\alpha_{11}+\alpha_{12}}{\alpha_{1}}\right) e^{\alpha_{1} t}+\frac{M_{1}}{\omega_{1}-\alpha_{1}}\left(e^{\omega_{1} t}-e^{\alpha_{1} t}\right) \\
& \leq M e^{\omega t}
\end{aligned}
$$

where we have employed (A.4). Furthermore, from (1.3) we obtain

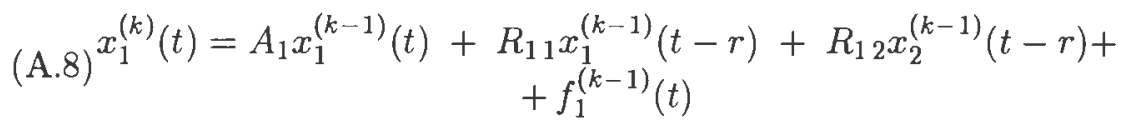

and using (A.5) this expression yields

$$
\begin{aligned}
\left\|x_{1}^{(k)}(t)\right\| & \leq M \alpha_{1} \omega^{k-1} e^{\omega t}+\left(\alpha_{11}+\alpha_{12}\right) \omega_{2}^{k-1} M_{2}+M_{1} \omega_{1}^{k-1} e^{\omega_{1} t} \\
& \leq \omega^{k-1}\left[\alpha_{1}+\left(\alpha_{11}+\alpha_{12}\right)\left(\frac{\omega_{2}}{\omega}\right)^{k-1}+\left(\frac{\omega_{1}}{\omega}\right)^{k-1}\right] e^{\omega t} M \\
& \leq M \omega^{k} e^{\omega t},
\end{aligned}
$$


which proves the assertion for $k \in \mathbb{N}$ and $t \in[0, r]$.

We assume now that the property holds for $t \in[0, k r]$. Let $t \in$ $(k r,(k+1) r\}$. Since

$$
x_{2}^{(k)}(t)=-\sum_{i=0}^{h-1} N^{i}\left[R_{21} x_{1}^{(i+k)}(t-r)+R_{22} x_{2}^{(i+k)}(t-r)+f_{2}^{(i+k)}(t)\right]
$$

then, from (A.6) we obtain

$$
\begin{aligned}
\left\|x_{2}^{(k)}(t)\right\| & \leq \sum_{i=0}^{h-1}\|N\|^{i}\left[\left(\alpha_{21}+\alpha_{22}\right) \omega^{i+k} e^{\omega(t-r)} M+M_{1} \omega_{1}^{i+k} e^{\omega_{1} t}\right] \\
& =\left(\sum_{i=0}^{h-1}\|N\|^{i}\left[\left(\alpha_{21}+\alpha_{22}\right) \omega^{i} e^{-\omega r}+\frac{M_{1}}{M} \omega_{1}^{i}\right]\right) M \omega^{k} e^{\omega t} \\
& \leq M \omega^{k} e^{\omega t} .
\end{aligned}
$$

On the other hand, the variation of constants formula implies that

$$
\begin{aligned}
& x_{1}(t)=e^{A_{1}(t-k r)} x_{1}(k r)+\int_{k r}^{t} e^{A_{1}(t-s)}\left[R_{11} x_{1}(s-r)+R_{12} x_{2}(s-r)+\right. \\
& \left.\quad+f_{1}(s)\right] d s .
\end{aligned}
$$

Hence

$$
\begin{aligned}
& \left\|x_{1}(t)\right\| \leq M e^{\alpha_{1}(t-k r)} e^{\omega k r}+\int_{k r}^{t} e^{\alpha_{1}(t-s)}\left[\left(\alpha_{11}+\alpha_{12}\right) M e^{\omega(s-r)}+\right. \\
& \left.+M_{1} e^{\omega_{1} s}\right] d s \\
& \leq M e^{\alpha_{1} t} e^{\left(\omega-\alpha_{1}\right) k r}+M e^{\alpha_{1} t \frac{\alpha_{11}+\alpha_{12}}{\omega-\alpha_{1}}}\left(e^{\left(\omega-\alpha_{1}\right) t}-e^{\left(\omega-\alpha_{1}\right) k r}\right) \\
& \frac{M_{1}}{\omega_{1}-\alpha_{1}} e^{\alpha_{1} t}\left(e^{\left(\omega_{1}-\alpha_{1}\right) t}-e^{\left(\omega_{1}-\alpha_{1}\right) k r}\right) \\
& \leq M e^{\omega t} \gamma(t)
\end{aligned}
$$

where we have introduced the function

$$
\begin{aligned}
\gamma(t):= & e^{\left(\alpha_{1}-\omega\right) t}\left[e^{\left(\omega-\alpha_{1}\right) k r}+\frac{\alpha_{11}+\alpha_{12}}{\omega-\alpha_{1}}\left(e^{\left(\omega-\alpha_{1}\right) t}-e^{\left(\omega-\alpha_{1}\right) k r}\right)\right. \\
& \left.+\frac{M_{1}}{M\left(\omega_{1}-\alpha_{1}\right)}\left(e^{\left(\omega_{1}-\alpha_{1}\right) t}-e^{\left(\omega_{1}-\alpha_{1}\right) k r}\right)\right],
\end{aligned}
$$


for $t \in[k r,(k+1) r]$. It is clear that $\gamma(k r)=1$. Moreover, it is follows from (A.7) that

$$
\begin{aligned}
\gamma \prime(t)= & e^{\left(\alpha_{1}-\omega\right) t}\left[\left(\alpha_{1}-\omega+\alpha_{11}+\alpha_{12}+\frac{M_{1}}{M} \frac{\omega-\alpha_{1}}{\omega_{1}-\alpha_{1}} e^{\left(\omega_{1}-\omega\right) k r}\right) e^{\left(\omega-\alpha_{1}\right) k r}-\right. \\
& \left.-\frac{M_{1}}{M} \frac{\omega-\omega_{1}}{\omega_{1}-\alpha_{1}} e^{\left(\omega_{1}-\alpha_{1}\right) t}\right]<0
\end{aligned}
$$

Thus, $\gamma(t)$ is a non increasing function on $[k r,(k+1) r]$ which proves that $\left\|x_{1}(t)\right\| \leq M e^{\omega t}$. Furthermore, from (A.8) and (A.5) we finally obtain

$$
\begin{aligned}
\left\|x_{1}^{(k)}(t)\right\| & \leq \alpha_{1} \omega^{k-1} M e^{\omega t}+\left(\alpha_{11}+\alpha_{12}\right) \omega^{k-1} M e^{\omega(t-r)}+M_{1} \omega_{1}^{k-1} e^{\omega_{1} t} \\
& \leq \omega^{k}\left[\frac{\alpha_{1}+\left(\alpha_{11}+\alpha_{12}\right) e^{-\omega r}}{\omega}+\frac{M_{1}}{M \omega}\left(\frac{\omega_{1}}{\omega}\right)^{k-1} e^{\left(\omega_{1}-\omega\right) t}\right] M e^{\omega t} \\
& \leq M \omega^{k} e^{\omega t}\left[\frac{\alpha_{1}+\left(\alpha_{11}+\alpha_{12}\right) e^{-\omega r}+1}{\omega}\right] \\
& \leq M \omega^{k} e^{\omega t} .
\end{aligned}
$$

This completes the proof.

The point of this result is that if $f(t)$ and $\varphi(\theta)$ are exponentially bounded then the solution $x(t)$ of problem (A.1)-(A.2) also is exponentially bounded.

\section{B. Appendix}

In this appendix we establish a stability property of retarded differential equations which is essential for our developments. We believe this property is well known. However, since we do not have found its proof in the literature, we have preferred, for reference, to include it here as a proposition. Next we use the terminology of [18]. In particular, we denote by $X(\cdot)$ the fundamental solution of the homogeneous retarded differential equation

$$
x^{\prime}(t)=A x(t)+B x(t-r)
$$

where $x(t) \in \boldsymbol{R}^{n}$ and $A$ and $B$ are $n \times n$ constant matrices. Further we denote by $x(\cdot, \varphi)$ the solution of the nonhomogeneous initial value problem

$$
\begin{aligned}
x^{\prime}(t) & =A x(t)+B x(t-r)+f(t) \\
x(\theta) & =\varphi(\theta), \quad-r \leq \theta \leq 0,
\end{aligned}
$$


where $\varphi$ is continuous on $[-r, 0]$ and $f$ is an appropriate function.

As consequence of the variation of constants formula $([18])$ and the asymptotic behavior of the Laplace transform we can establish.

Proposition B.1. If (B.1) is asymptotically stable and $f:[0, \infty) \rightarrow \boldsymbol{R}^{n}$ is a function of exponential type such that $f(t)$ converges to $f_{0}$ as $t \rightarrow \infty$, then $x(t, \varphi) \rightarrow-(A+B)^{-1} f_{0}$ as $t \rightarrow \infty$ for all $\varphi$.

Proof. From the variation of constants formula ([18], Theorem 1.6.1) it follows that

$$
x(t, \varphi)=y(t)+\int_{0}^{t} X(t-s) f(s) d s
$$

where $y$ denotes the solution of (B.1) with initial condition $\varphi$. Since $y(t) \rightarrow$ 0 as $t \rightarrow \infty$ only remains to prove that

$$
u(t):=\int_{0}^{t} X(t-s) f(s) d s
$$

converges to $-(A+B)^{-1} f_{0}$ as $t$ goes to infinity. Using the Cauchy criteria we easily derive that $u(t)$ is convergent as $t \rightarrow \infty$. On the other hand, if $\mathcal{L}$ stands for the Laplace transform, applying theorem 34.2 and theorem 34.3 in [9] we can write

$$
\begin{aligned}
\lim _{t \rightarrow \infty} u(t) & =\lim _{\lambda \rightarrow 0} \lambda \mathcal{L} u(\lambda) \\
& =\lim _{\lambda \rightarrow 0} \lambda\left(\lambda I-A-e^{-\lambda r} B\right)^{-1} \mathcal{L} f(\lambda) \\
& =-(A+B)^{-1} f_{0} .
\end{aligned}
$$

\section{Appendix}

In this appendix we collect some simple results of purely algebraic nature, which have been used in the previous section.

Lemma C.1. Assume that $(E, A, C)$ is observable. Let $L:=\widetilde{E}-G C$ and set

$$
P^{-1} L P:=\left[\begin{array}{ll}
L_{1,1} & L_{1,2} \\
L_{2,1} & L_{2,2}
\end{array}\right] .
$$

Then we can select the matrix $G$ so that both $L$ and $L_{1,1}$ to be invertible matrices and $\operatorname{det}((s-\mu) L-I) \neq 0$, for $\operatorname{Re}(s) \geq 0$. 
Proof. In fact, with the matrix $P^{-1} G:=\left[\begin{array}{c}C_{1} \\ 0\end{array}\right]$, from the definution of $L$ and $\widetilde{E}$ and using the transformation of coordinates we can write

$$
\begin{aligned}
P^{-1} L P & =P^{-1}(A-\mu E)^{-1} E P-P^{-1} G C P \\
& =P^{1}(A-\mu E)^{-1} Q^{-1} Q E P-P^{-1} G C P \\
& =(Q(A-\mu E) P)^{-1} Q E P-P^{-1} G C P \\
& =\left[\begin{array}{cc}
A_{1}-\mu I & 0 \\
0 & I-\mu N
\end{array}\right]^{-1}\left[\begin{array}{cc}
I & 0 \\
0 & N
\end{array}\right]-P^{-1} G C P \\
& =\left[\begin{array}{cc}
\left(A_{1}-\mu I\right)^{-1} & 0 \\
0 & (I-\mu N)^{-1}
\end{array}\right]\left[\begin{array}{cc}
I & 0 \\
0 & N
\end{array}\right]-\left[\begin{array}{cc}
G_{1} C_{1} & G_{1} C_{2} \\
0 & 0
\end{array}\right] \\
& =\left[\begin{array}{cc}
\left(A_{1}-\mu I\right)^{-1}-G_{1} C_{1} & -G_{1} C_{2} \\
0 & (I-\mu N)^{-1} N
\end{array}\right] .
\end{aligned}
$$

Thus $L_{1,1}=\left(A_{1}-\mu I\right)^{-1}-G_{1} C_{1} ; \quad L_{1,2}=-G_{1} C_{2} ; \quad L_{2,1}=0 \quad$ and $L_{2,2}=(I-\mu N)^{-1} N$, which implies that

$$
\operatorname{det}((s-\mu) L-I)=\stackrel{+}{-} \operatorname{det}\left((s-\mu) L_{1,1}-I\right) .
$$

Since $\left(A_{1}, C_{1}\right)$ is observable we can choose $G_{1}$ so that $L_{1,1}$ is invertible and the solutions of the equation $\operatorname{det}\left((s-\mu) L_{1,1}-I\right)=0$ are located in $\operatorname{Re}(s)<0$. This completes the proof.

Next we establish the existence of a suitable coordinate transformation to reduce a singular system in block form to its standard form.

Lemma C.2. Let $N, H$ and $R$ be $n \times n, m \times m$ and $n \times m$ matrices, respectively, such that $N$ is nilpotent. Then there exists a $n \times m$ matrix $X$ which satisfies the conditions

$$
\left[\begin{array}{cc}
I_{m} & 0 \\
-N X & I_{n}
\end{array}\right]\left[\begin{array}{cc}
I_{m} & 0 \\
0 & N
\end{array}\right]\left[\begin{array}{cc}
I_{m} & 0 \\
X & I_{n}
\end{array}\right]=\left[\begin{array}{cc}
I_{m} & 0 \\
0 & N
\end{array}\right]
$$

and

$$
\left[\begin{array}{cc}
I_{m} & 0 \\
-N X & I_{n}
\end{array}\right]\left[\begin{array}{cc}
H & 0 \\
R & I_{n}
\end{array}\right]\left[\begin{array}{cc}
I_{m} & 0 \\
X & I_{n}
\end{array}\right]=\left[\begin{array}{cc}
H & 0 \\
0 & I_{n}
\end{array}\right]
$$


Proof. Let $h$ be the index of $N$. We define $X$ as the matrix

$$
X:=-\sum_{i=0}^{h-1} N^{i} R H^{i} .
$$

It is clear from this definition that $N X H=R+X$ and that, in turn, relations (C.1) and (C.2) are easy consequences of this property.

\section{References}

[1] Bellman, R. and Cooke, K., Differential-Difference Equations. Academic Press, New York, (1963).

[2] Bhat, K. P. M. and Koivo, H. N., An Observer Theory for Time-Delay Systems. IEEE Trans. Aut. Contr. 21, pp. 266-269, (1976).

[3] Brierley, S. D.; Chiasson, J. N.; Lee, E. B. and Zak, S. H., On Stability Independent of Delay for Linear Systems. IEEE Trans. Aut. Contr. 27 (1), pp. 252-254, (1982).

[4] Campbell, S. L., Singular systems of differential equations. Pitman Advanced Publishing Program, London, (1980).

[5] Campbell, S. L., Singular systems of differential equations II. Pitman Advanced Publishing Program, London, (1982).

[6] Chen, B-S.; Wang, S-S. and Lu, H-C., Stabilization of time-delay systems containing saturating actuators. Int. J. Control 47 (3), pp. $867-$ $881,(1988)$.

[7] Dai, L., Singular control systems. Lect. Notes in Control and Information Sciences, 118. Springer Verlag, Berlin, (1989).

[8] Datko, R., Remarks Concerning the Asymptotic Stability and Stabilization of Linear Delay Differential Equations. J. Math. Anal. Appl., 111, pp. 571-584, (1985).

[9] Doetsh, G., Introduction to the Theory and Application of the Laplace Transformation. Springer-Verlag, Berlin, (1974). 
[10] Emre, E., Regulation of linear systems over rings by dynamic output feedback. Systems and Control Letters 3, pp. 57-62, (1983).

[11] Emre, E. and Khargonekar, P. P., Regulation of Split Linear Systems Over Rings: Coefficient-Assignment and Observers. IEEE Trans. Aut. Contr. 27 (1), pp. 104-113, (1982).

[12] Emre, E. and Knowles, G. J., Control of Linear Systems with Fixed Noncommensurate Point delays. IEEE Trans. Aut. Contr. 29(12), pp. 1083-1090, (1984).

[13] Fahmy, M. M. and O'Reilly, J., Observers for descriptor systems. Int. J. Control, 49, pp. 2013-2028, (1989).

[14] Fairman, F. W. and Kumar, A., Delayless Observers for Systems with Delay. IEEE Trans. Aut. Contr. 31(3), pp. 258-259, (1986).

[15] Fiagbedzi, Y. A. and Pearson, A. E., Feedback Stabilization of Linear Autonomous Time Lag Systems. IEEE Trans. Aut. Contr. 31(9), pp. 847-855, (1986).

[16] Fuchs, A.; Lovass-Nagy, V. and Mukundan, R., Output regulator problem of time-invariant discrete-time descriptor systems, Int. J. Control, 46, pp. 2065-2074, (1987).

[17] Halanay, A., Differential Equations. Academic Press, New York, (1966).

[18] Hale, J. and Verduyn Lunel, S. M., Introduction to Functional Differential Equations. Springer Verlag, New York, (1993).

[19] Hautus, M. L. J., Controllability and Observability conditions of linear autonomous systems. Indag. Math., 31, pp. 443-448, (1969).

[20] Henríquez, H., Regulator problem for linear distributed control systems with delays in outputs Lect. Notes in Pure and Applied Maths. 155, pp. 259-273. Marcel Dekker, New York, (1994).

[21] Kamen, E. W., On the Relationship Between Zero Criteria for TwoVariable Polynomials and Asymptotic Stability of Delay Differential Equations. IEEE Trans. Aut. Contr. 25(5), pp. 983-984, (1980).

[22] Kamen, E. W., Linear Systems with Commensurate Time Delays: Stability and Stabilization Independent of Delay. IEEE Trans. Aut. Contr. 27(2), pp. 367-375, (1982). 
[23] Kamen, E. W., Correction to "Linear Systems with Commensurate Time Delays: Stability and Stabilization Independent of Delay". IEEE Trans. Aut. Contr. 28(2), pp. 248-249, (1983).

[24] Kamen, E. W.; Khargonekar, P. P. and Tannenbaum, A., Stabilization of Time-Delay Systems Using Finite-Dimensional Compensators. IEEE Trans. Aut. Contr. 30(1), pp. 75-78, (1985).

[25] Kamen, E. W.; Khargonekar, P. P. and Tannenbaum, A., Proper stable Bezout factorization and feedback control of linear time-delay systems. Int. J. Control 43(3), pp. 837-857, (1986).

[26] Klamka, J., Observer for linear feedback control of systems with distributed delays in controls and outputs. Systems and Control Letters, 1(5), pp. 326-331, (1982).

[27] Koumboulis, F. N. and Paraskevopoulos, P. N., On the pole assignment of generalized state space systems via state feedback, IEE ProceedingsD, 139, pp. 106-108, (1992).

[28] Lewis, R. M. and Anderson, B., Necessary and Sufficient Conditions for Delay-Independent Stability of Linear Autonomous Systems. IEEE Trans. Aut. Contr. 25(4), pp. 735-739, (1980).

[29] Manitius, A. Z. and Olbrot, A. W., Finite Spectrum Assignment Problem for Systems with Delays. IEEE Trans. Aut. Contr. 24(4), pp. 541553, (1979).

[30] Minamide, N.; Arii, N. and Uetake, Y., 1989, Design of observers for descriptor systems using a descriptor standard form. Int. J. Control, 50, pp. 2141-2149, (1989).

[31] Olbrot, A. W., Stabilizability, Detectability, and Spectrum Assignment for Linear Autonomous Systems with General Time Delays. IEEE Trans. Aut. Contr., 23, pp. 887-890, (1978).

[32] O'Reilly, J., Observers for linear systems. Academic Press, London, (1983).

[33] Pandolfi, L., Feedback stabilization of functional differential equations. Boll. Un. Mat. Ital., 12, pp. 626-635, (1975). 
[34] Paraskevopoulus, P. N. and Koumboulis, F. N., Decoupling and pole assignment in generalized state space systems. IEE Proceedings- $D$, 138(6), pp. 547-560, (1991).

[35] Paraskevopoulos, P. N. and Koumboulis, F. N., Unifyng approach to observers for regular and singular systems. IEE Proceedings- $D, 138(6)$, pp. 561-572, (1991).

[36] Paraskevopoulos, P. N.; Koumboulis, F. N.; Tzierakis, K. G. and Panagiotakis, G. E., Observer design for generalized state space systems with unknown inputs. Systems and Control Letters, 18, pp. 309-321, (1992).

[37] Salamon, D., Observers and Duality Between Observation and State Feedback for Time Delay Systems. IEEE Trans. Aut. Contr. 25(6), pp. 1187-1192, (1980).

[38] Schumacher, J. M., A direct approach to compensator design for distributed parameter systems. SIAM J. Contr. Optimiz. 21(6), pp. 823-836, (1983).

[39] Uetake, Y., Pole assignment and observer design for continuous descriptor systems. Int. J. Control, 50, pp. 89-96, (1989).

[40] Wang, Q-G.; Sun, Y. X. and Zhou, C. H., Finite spectrum assignment for multivariable delay systems in the frequency domain. Int. $J$. Control 47(3), pp. 729-734, (1988).

[41] Watanabe, K. and Ito, M., An observer for linear feedback control laws of multivariable systems with multiple delays in controls and outputs. Systems and Control Letters, 1(1), pp. 54-59, (1981).

[42] Watanabe, K.; Ito, M. and Kaneko, M., Finite spectrum assignment problem for systems with multiple commensurate delays in state variables. Int. J. Control 38(5), pp. 913-926, (1983).

[43] Watanabe, K.; Ito, M. and Kaneko, M., Finite spectrum assignment problem for systems with multiple commensurate delays in states and control. Int. J. Control 39(5), pp. 1073-1082, (1984).

[44] Watanabe, K. and Ouchi, T., An observer of systems with delays in state variables. Int. J. Control 41(1), pp. 217-229, (1985). 
[45] Watanabe, K., Finite Spectrum Assignment and Observer for Multivariable Systems with Commensurate Delays. IEEE Trans. Aut. Contr. 31(6), pp. 543-550, (1986).

[46] Wonham, W. M., Linear multivariable control: A geometric approach. Springer Verlag, Berlin, (1979).

[47] Yang, C-W. and Tan, H-L., Observer design for singular systems with unknown inputs. Int. J. Control, 49(6), pp. 1937-1946, (1989).

Received : November 13, 1997.

\section{Hernán Henriquez}

Departamento de Matemáticas

Universidad de Santiago de Chile

Casilla 307

Correo 2

Santiago

Chile

$\mathrm{y}$

Genaro Castillo

Departamento de Matemáticas

Universidad de Talca

Campus Lircay

Talca

Chile 